\title{
A Hamiltonian fluid-kinetic model for a two-species non-neutral plasma
}

\author{
E. Tassi ${ }^{1}$, M. Romé ${ }^{2}$ and C. Chandre ${ }^{1}$ \\ 1 Aix-Marseille Université, Université de Toulon, \\ CNRS CPT UMR 7332, 13288 Marseille, France \\ 2 INFN Sezione di Milano and Dipartimento di Fisica, \\ Università degli Studi di Milano, Via Celoria 16, I-20133 Milano, Italy
}

\begin{abstract}
A model for describing the dynamics of a pure electron plasma in the presence of a population of massive charged particles is presented. The model couples the fluid dynamics of the pure electron plasma with the dynamics of the massive particle population, the latter being treated kinetically. The model is shown to possess a noncanonical Hamiltonian structure and to preserve invariants analogous to those of the two-dimensional (2D) Euler equation for an incompressible inviscid fluid, and of the Vlasov equation. The Hamiltonian structure of the model is used to derive a set of stability conditions for rotating coherent structures of the two-species system, in the case of negatively charged massive particles. According to these conditions, stability is attained if both the equilibrium distribution function of the kinetic species and the equilibrium density of the electron fluid are monotonically decreasing functions of the corresponding single-particle energies in the rotating frame. For radially confined equilibria near the axis, the stability condition corresponds to the existence of a finite interval of rotation frequencies for the reference frame, with the upper bound determined by the presence of the kinetic population.
\end{abstract}

The dynamics of single-species non-neutral plasmas has been studied extensively in the literature $[1,2]$. We consider here the behavior of a multi-species non-neutral plasma, and more specifically an electron plasma, subject to a uniform and constant magnetic field, and in the presence of a population of massive negatively charged particles.

An example of a multispecies negatively charged nonneutral plasma is the electron-antiproton plasma used as an intermediate step in the production of trapped antihydrogen [3]. The experimental investigation of the dynamics of a non-neutral complex plasma, and in particular a plasma of electrons contaminated with a small fraction of sub-micrometric charged dust particles is the subject of the "DuEl" project $[4,5]$.

Under conditions of strong magnetization of the electron component, which are typical of the experiments performed on non-neutral plasmas confined in PenningMalmberg traps, a cold fluid description of the electron dynamics is adopted, in which electron inertia as well as temperature effects are neglected [1]. On the other hand, the dynamics of massive charged particles occurs on slower time scales, since their charge to mass ratio is orders of magnitude smaller than the corresponding ratio of the electrons. Moreover, at relatively low magnetic field strengths regimes can be found where ions are only weakly magnetized.

Under these circumstances, the simplest model to describe these multi-species systems consists of a hybrid system which couples, through Poisson's equation, a kinetic equation for the massive species with a fluid equation for the electron guiding centers. A further simplification that can be adopted for these systems consists of considering a 2D dynamics. For pure electron plasmas in Penning-Malmberg traps the high-frequency longitudinal electron motion can be averaged out for a wide range of operational regimes, and the electron plasma dynamics becomes isomorphic to that of a $2 \mathrm{D}$ incompressible inviscid fluid, where the fluid vorticity corresponds to the electron density and the fluid stream function to the electrostatic potential $[6,7]$. A 2D multi-fluid model turns out to be appropriate also for a cold non-relativistic electronion system at high magnetic field strengths [1], while a kinetic treatment for the dynamics of the ion population is required at moderate values of the confining field. The hybrid model we present here can obviously be generalized to account for multiple negative ion species treated kinetically. Furthermore, the model can also be applied to the case of a positron plasma in the presence of kinetic populations of positive ions (see, e.g., Ref. [8]).

On the basis of the above assumptions we consider a plasma contained in a cylindrical domain of radius $a$ and length $L_{z}$ and we use cylindrical coordinates $(r, \theta, z)$, with $z$ along the constant and uniform magnetic field, $\mathbf{B}=B_{0} \hat{z}$. Our model equations are then given by

$$
\begin{array}{r}
\frac{\partial n_{e}}{\partial t}+\left[\phi, n_{e}\right]=0 \\
\frac{\partial f}{\partial t}+\mathbf{v} \cdot \nabla f+\left[\frac{q_{I}}{M}\left(-\nabla \phi+\frac{\mathbf{v}}{c} \times \mathbf{B}\right)+\mathbf{g}\right] \cdot \frac{\partial f}{\partial \mathbf{v}}=0 \\
\Delta \phi=4 \pi\left(e n_{e}-q_{I} \int d^{2} v f\right) .
\end{array}
$$

In the above system $n_{e}$ indicates the guiding center electron density, $f\left(r, \theta, v_{r}, v_{\theta}\right)$ is the distribution function of the massive particle population (integrated over $v_{z}$ ), $\phi$ is the electrostatic potential, $-e$ the electron charge, $q_{I}<0$ the charge of the massive particle, $M$ its mass, $\mathbf{g}=-g(\sin \theta \hat{r}+\cos \theta \hat{\theta})$ the acceleration due to gravity (the latter may become non-negligible for heavy ions), $c$ the speed of light and $d^{2} v=d v_{r} d v_{\theta}$ the surface element in velocity space. Finally, we defined the bracket $[f, h]$ between two functions $f$ and $h$ of the spatial variables $(r, \theta)$ as $[f, h]=\left(c / B_{0} r\right)\left(\partial_{r} f \partial_{\theta} h-\partial_{\theta} f \partial_{r} h\right)$. 
Equation (1) represents a continuity equation for the electron guiding center density, which is advected by the incompressible $\mathbf{E} \times \mathbf{B}$ flow. Equation (2) is a Vlasov equation that governs the evolution of the massive particle population, whereas Eq. (3) is the Poisson's equation determining the electrostatic potential.

Equations (1)-(3) form a Hamiltonian system assuming that boundary terms vanish when integrating by parts. The Hamiltonian functional $H$ and the Poisson bracket $\{$,$\} are given by$

$$
\begin{aligned}
& H\left(n_{e}, f\right)=\int d A d^{2} v\left(M \frac{v_{r}^{2}+v_{\theta}^{2}}{2}+M g r \sin \theta\right) f \\
& -\frac{1}{8 \pi} \int d A \phi \Delta \phi,
\end{aligned}
$$

and

$$
\{F, G\}=\int d A \frac{n_{e}}{e}\left[F_{n_{e}}, G_{n_{e}}\right]+\int d A d^{2} v f\left[F_{f}, G_{f}\right]_{v},
$$

respectively. In Eq. (5), the subscripts of $F$ and $G$ indicate functional derivatives, whereas $d A=r d r d \theta$ is the surface element. The bracket $[f, h]_{v}$ in Eq. (5) is defined by

$$
\begin{aligned}
& {[f, h]_{v}=\frac{1}{M}\left[\frac{\partial f}{\partial r} \frac{\partial h}{\partial v_{r}}-\frac{\partial f}{\partial v_{r}} \frac{\partial h}{\partial r}+\frac{1}{r} \frac{\partial f}{\partial \theta} \frac{\partial h}{\partial v_{\theta}}-\frac{1}{r} \frac{\partial f}{\partial v_{\theta}} \frac{\partial h}{\partial \theta}\right.} \\
& \left.+\left(\frac{v_{\theta}}{r}+\frac{q_{I} B_{0}}{M c}\right)\left(\frac{\partial f}{\partial v_{r}} \frac{\partial h}{\partial v_{\theta}}-\frac{\partial f}{\partial v_{\theta}} \frac{\partial h}{\partial v_{r}}\right)\right] .
\end{aligned}
$$

Note that in Eq. (4) the Hamiltonian $H$ is defined as an energy per unit length. It can be easily verified that the dynamical equations (1) and (2) are obtained from the expression $\partial \chi / \partial t=\{\chi, H\}$ which governs the evolution of a generic Hamiltonian system, by replacing $\chi$ with $n_{e}$ and $f$, the Hamiltonian functional with Eq. (4), the Poisson bracket with Eq. (5), and making use of the Poisson's equation (3). Note that the bracket in Eq. (5) is the direct sum of two independent Poisson brackets, namely those for the incompressible 2D Euler equation [9] and for the Vlasov equation [10, 11]. Consequently it qualifies as a Poisson bracket, and in particular it satisfies the Jacobi identity.

This bracket possesses two independent families of Casimir invariants, that is functionals $F$, such that $\{F, C\}=0$ for all functionals $C\left(n_{e}, f\right)$. These families are given by

$$
C_{1}=\int d A \mathcal{F}\left(n_{e}\right), \quad C_{2}=\int d A d^{2} v \mathcal{G}(f),
$$

with $\mathcal{F}$ and $\mathcal{G}$ arbitrary functions. The invariants include, as particular cases, the familiar invariants of the $2 \mathrm{D} \mathrm{Eu}-$ ler incompressible equations, such as the enstrophy, and those of the Vlasov equation, such as the entropy and the total number of particles.

We remark that further examples of Hamiltonian hybrid fluid-kinetic models, accounting for coupling via the current density, or via the pressure, have been discussed in Refs. [12-14].

The Hamiltonian structure of the model is exploited to obtain stability conditions for equilibria of the hybrid system (1)-(3) using the Energy-Casimir method (see, e.g., Refs. [15-18]). We restrict the analysis to the case of two species rotating azimuthally with the same uniform and constant frequency $\omega$. Equilibria of interest are then those which are stationary in the rotating frame. In order to apply the Energy-Casimir method, the Hamiltonian structure of the model equations in the rotating frame is required. A convenient way to accomplish this is to look for a new set of field variables, in terms of which the Poisson bracket keeps the same form, whereas additional contributions accounting for the change in the reference frame appear in the Hamiltonian. The advantage of this approach is that the Casimir invariants required for the application of the method are already available, since they only depend on the Poisson bracket. However, this procedure is in general non trivial, because the change to the rotating frame $\theta^{\prime}=\theta-\omega t$ is performed at the level of the single-particle phase space, whereas the Poisson bracket (5) is defined on the algebra of functionals of the field variables.

Since the transformation of coordinates to the rotating frame is time-dependent, it is necessary to introduce a new evolution parameter and extend the algebra of observables to the set of functionals $U\left(n_{e}, f, t, k\right)$, where $t$ is the time dynamical variable and $k$ its canonically conjugate variable with respect to the extended Poisson bracket

$$
\{F, G\}_{t}=\{F, G\}+\frac{\partial F}{\partial t} \frac{\partial G}{\partial k}-\frac{\partial G}{\partial t} \frac{\partial F}{\partial k} .
$$

Consequently, we have to consider in the extended phase space the new Hamiltonian $H_{t}=H+k_{\dot{\tilde{f}}}$ We then look for a new set of dynamical variables $\left(\tilde{n}_{e}, \tilde{f}, \tilde{t}, \tilde{k}\right)$ such that

$$
\begin{aligned}
& \tilde{n}_{e}\left(r, \theta^{\prime}\right)=n_{e}\left(r, \theta^{\prime}+\omega t\right), \quad \tilde{k}=k+W\left(n_{e}, f\right), \\
& \tilde{f}\left(r, \theta^{\prime}, v_{r}, v_{\theta}\right)=f\left(r, \theta^{\prime}+\omega t, v_{r}, v_{\theta}\right), \quad \tilde{t}=t,
\end{aligned}
$$

where $W\left(n_{e}, f\right)$ is a functional determined by the constraint that the bracket (5) is invariant with respect to the change of variables. The mapping (9) determines the following transformation for the derivatives of the functionals:

$$
\begin{aligned}
& F_{\chi}=\tilde{F}_{\tilde{\chi}}+W_{\chi} \frac{\partial \tilde{F}}{\partial \tilde{k}}, \quad \frac{\partial F}{\partial k}=\frac{\partial \tilde{F}}{\partial \tilde{k}}, \\
& \frac{\partial F}{\partial t}=\frac{\partial \tilde{F}}{\partial \tilde{t}}+\omega\left(\int d A \frac{\partial \tilde{n}_{e}}{\partial \theta^{\prime}} \tilde{F}_{\tilde{n}_{e}}+\int d A d^{2} v \frac{\partial \tilde{f}}{\partial \theta^{\prime}} \tilde{F}_{\tilde{f}}\right),
\end{aligned}
$$

where $\chi$ is either $n_{e}$ or $f$. Inserting the expressions (9)(10) into Eq. (5), the bracket turns out to be invariant if $W=\omega P_{\theta e}+\omega P_{\theta I}$, where $P_{\theta e}=-\int d A\left(e B_{0} / 2 c\right) r^{2} n_{e}$ and $P_{\theta I}=\int d A d^{2} v\left[\left(q_{I} B_{0} / 2 c\right) r^{2}+M r v_{\theta}\right] f$. As expected, $P_{\theta e}$ 
and $P_{\theta I}$ correspond to the total canonical angular momenta of massless electrons and of the ions, respectively.

We restrict the stability analysis to equilibria which are independent on $\theta$. Consequently, from now on we consider physical situations where the gravitational force may be neglected. This approximation also removes the time dependence of the Hamiltonian in the new reference frame. The assumption is valid also for heavy ions but under microgravity conditions [19].

According to the Energy-Casimir method, equilibria of the system correspond to extremals of the free energy functional $F$ given by the linear combination of the Hamiltonian with the Casimirs of the Poisson bracket. Since we consider now a time-independent Hamiltonian in the new reference frame, we omit the contribution of $k$. For ease of notation we also drop the tilde symbol for the new variables. Stationary equilibria in the rotating frame therefore correspond to extremals of [1]

$$
F=H-\omega P_{\theta e}-\omega P_{\theta I}+C_{1}+C_{2},
$$

i.e., to solutions of

$$
F_{n_{e}}=0, \quad F_{f}=0 .
$$

These are given by

$$
\mathcal{F}^{\prime}\left(n_{e}\right)=-h_{e}, \quad \mathcal{G}^{\prime}(f)=-h_{I},
$$

where the prime denotes the derivative with respect to the argument of the function and where $h_{e}=-e \phi+$ $\omega\left(e B_{0} / 2 c\right) r^{2}$ and $h_{I}=M v^{2} / 2+q_{I} \phi-\omega\left[\left(q_{I} B_{0} / 2 c\right) r^{2}+\right.$ $\left.M r v_{\theta}\right]$ indicate the single particle energies in the rotating frame of massless electrons and of the contaminant particles, respectively. Assuming that the arbitrary functions $\mathcal{F}^{\prime}$ and $\mathcal{G}^{\prime}$ are invertible, the relations (13) imply that, at equilibrium, $n_{e}=n_{e e q}\left(h_{e}\right)$ and $f=f_{e q}\left(h_{I}\right)$, where $n_{e e q}=\left(-\mathcal{F}^{\prime}\right)^{-1}$ and $f_{e q}=\left(-\mathcal{G}^{\prime}\right)^{-1}$.

According to the Energy-Casimir method, energy stability (also called formal stability) of the equilibria obtained by setting $\delta F=0$, is guaranteed if $\delta^{2} F$, evaluated for such equilibria, has a definite sign for all field variations. We recall that this type of stability implies linear stability (see, e.g. Refs. [17, 18])

We consider field variations $\delta n_{e}$ and $\delta f$ periodic with respect to $\theta$ and vanishing at $r=0$ and $r=a$. We also assume that $\delta f$ tends to zero for $v_{r}$ and $v_{\theta}$ going to infinity. The second variation $\delta^{2} F$ can then be computed as

$$
\begin{aligned}
& \delta^{2} F\left(n_{e e q}, f_{e q}\right)=\int d A\left(\frac{|\nabla \delta \phi|^{2}}{8 \pi}+\mathcal{F}^{\prime \prime}\left(n_{e e q}\right)\left|\delta n_{e}\right|^{2}\right) \\
& +\int d A d^{2} v \mathcal{G}^{\prime \prime}\left(f_{e q}\right)|\delta f|^{2}
\end{aligned}
$$

where $\Delta \delta \phi=4 \pi\left(e \delta n_{e}-q_{I} \int d^{2} v \delta f\right)$. The second variation of the free energy functional is then positive if $\mathcal{F}^{\prime \prime}\left(n_{e e q}\right) \geq 0$ and $\mathcal{G}^{\prime \prime}\left(f_{e q}\right) \geq 0$.
Using Eq. (13) and the relations $n_{e e q}=\left(-\mathcal{F}^{\prime}\right)^{-1}$ and $f_{e q}=\left(-\mathcal{G}^{\prime}\right)^{-1}$, a set of stability conditions is therefore given by

$$
\frac{d n_{e e q}}{d h_{e}} \leq 0, \quad \frac{d f_{e q}}{d h_{I}} \leq 0 .
$$

In the limit of single-species plasmas, the inequalities (14) separately correspond to the stability condition for a cold pure electron plasma equilibrium in the fluid guidingcenter description and for a kinetic equilibrium of a population of massive ions, respectively [1, 20, 21].

Defining $\bar{n}_{e}(r)=n_{e e q}\left(h_{e}\right), \bar{f}\left(r, v_{r}, v_{\theta}\right)=f_{e q}\left(h_{I}\right)$ and $\bar{n}_{I}(r)=\int d^{2} v \bar{f}\left(r, v_{r}, v_{\theta}\right)$. Conditions (14) imply for confined equilibria with monotonically decreasing density profiles $\left(\bar{n}_{e}^{\prime}(r) \leq 0, \bar{n}_{I}^{\prime}(r) \leq 0\right)$ that

$$
\omega \geq \frac{4 \pi}{r^{2}} \frac{c}{B_{0}}\left[\int_{0}^{r} d s s\left(e \bar{n}_{e}(s)-q_{I} \bar{n}_{I}(s)\right)\right],
$$

and

$$
\omega_{-} \leq \omega \leq \omega_{+}
$$

respectively, with

$\omega_{ \pm}=\frac{\omega_{c i}}{2}\left[1 \pm\left(1+\frac{16 \pi q_{I}}{M \omega_{c i}^{2} r^{2}} \int_{0}^{r} d s s\left(e \bar{n}_{e}(s)-q_{I} \bar{n}_{I}(s)\right)\right)^{1 / 2}\right]$

where $\omega_{c i}=\left|q_{I}\right| B_{0} / M c$ is the ion cyclotron frequency and use has been made of the relation $\phi^{\prime}(r)=$ $(4 \pi / r) \int_{0}^{r} d s\left(e \bar{n}_{e}(s)-q_{I} \bar{n}_{I}(s)\right)$, obtained from Eq. (3) imposing the condition $\phi^{\prime}(0)=0$.

Close to the axis (so that $\bar{n}_{e}(r) \approx \bar{n}_{e}(0)$ and $\bar{n}_{I}(r) \approx$ $\left.\bar{n}_{I}(0)\right)$, the reality condition for $\omega_{ \pm}$corresponds to the self-field parameter $4\left(\omega_{D I}+\omega_{D e}\right) / \omega_{c i}$ to be less than unity, with $\omega_{D I}=2 \pi\left|q_{I}\right| c \bar{n}_{I}(0) / B_{0}$ and $\omega_{D e}=$ $2 \pi e c \bar{n}_{e}(0) / B_{0}$, the ion and electron diocotron frequencies, respectively. In particular, if $4\left(\omega_{D I}+\omega_{D e}\right) / \omega_{c i} \ll 1$, the conditions (15) and (16) reduce, at the leading order, to

$$
\begin{array}{r}
\omega \geq \omega_{D e}+\omega_{D I}, \\
\omega_{D e}+\omega_{D I} \leq \omega \leq \omega_{c i} .
\end{array}
$$

Clearly, the condition (18) is implied by the condition (19). The latter relation identifies a finite range of frequencies $\omega$ for which the stability condition can be satisfied for confined equilibria. When the ion species acts as a small contamination of a pure electron plasma, the condition $4\left(\omega_{D I}+\omega_{D e}\right) / \omega_{c i} \ll 1$ reduces to $4 \omega_{D e} \ll \omega_{c i}$, i.e., $\bar{n}_{e}(0) \ll(1 / 8 \pi)\left|q_{I}\right| B_{0}^{2} / e M c^{2}$. This corresponds to a modified Brillouin electron density limit, which depends also on the charge and the mass of the contaminant species. In the opposite case, $\bar{n}_{e}(0)=0$, the usual Brillouin density limit for the negative ion species is retrieved, $\bar{n}_{I}(0) \ll(1 / 8 \pi) B_{0}^{2} / M c^{2}$.

An important example of stable confined equilibrium can be obtained from an electron density and an ion distribution function corresponding 
to $n_{e e q}\left(h_{e}\right)=\bar{n}_{e}(0) \exp \left(-h_{e} / \kappa T\right)$ and $f_{e q}\left(h_{I}\right)=$ $\left(\bar{n}_{I}(0) M / 2 \pi \kappa T\right) \exp \left(-h_{I} / \kappa T\right)$, respectively, where $\kappa$ denotes the Boltzmann constant and $T$ is a constant temperature. For this case, the conditions (14) are clearly satisfied and the confinement condition (19) follows requiring $\bar{n}_{I}^{\prime}(r)=\left(\bar{n}_{I}(0) / \kappa T\right)\left(-\omega^{2} M r+\omega\left|q_{I}\right| B_{0} r / c-\right.$ $\left.\left|q_{I}\right| \phi^{\prime}\right) \exp \left(-h_{I} / \kappa T\right)$ to be negative. The stability and confinement of non-neutral plasmas in thermal equilibrium have been discussed in Ref. [22] and confinement conditions analogous to Eq. (19) for kinetic multi-species thermal plasmas have been described in Ref. [1]. In the hybrid model presented here, where the electron species is treated as a massless fluid, for stable equilibria with small self-field parameter the confinement condition for the negative ion species is sufficient to guarantee the con- finement of both species.

This work was supported by the European Community under the contract of Association between EURATOM, CEA, and the French Research Federation for fusion studies. The views and opinions expressed herein do not necessarily reflect those of the European Commission. Financial support was also received from the Agence Nationale de la Recherche (ANR GYPSI) and from the CNRS PEPS project GEOPLASMA. This work was also partially supported by the Italian Ministry for University and Research (MIUR) "PRIN 2009" funds (Grant No. 20092YP7EY). E. T. acknowledges fruitful discussions with Eric Soccorsi as well as useful interactions with Cesare Tronci and Phil Morrison about Hamiltonian hybrid models.
[1] R. C. Davidson, An Introduction to the Physics of Nonneutral Plasmas (Addison-Wesley, Redwood City, 1990).

[2] T. M. O’Neil, Phys. Today 52, 24 (1999).

[3] G. B. Andresen, M. D. Ashkezari, M. Baquero-Ruiz, W. Bertsche, P. D. Bowe, E. Butler, C. L. Cesar, S. Chapman, M. Charlton, A. Deller, S. Eriksson, J. Fajans, T. Friesen, M. C. Fujiwara, D. R. Gill, A. Gutierrez, J. S. Hangst, W. N. Hardy, M. E. Hayden, A. J. Humphries, R. Hydomako, S. Jonsell, N. Madsen, S. Menary, P. Nolan, A. Olin, A. Povilus, P. Pusa, F. Robicheaux, E. Sarid, D. M. Silveira, C. So, J. W. Storey, R. I. Thompson, D. P. van der Werf, J. S. Wurtele, and Y. Yamazaki (ALPHA Collaboration), Phys. Rev. Lett. 106, 145001 (2011).

[4] M. Romé, F. Cavaliere, M. Cavenago, M. Ikram, F. Lepreti, G. Maero, B. Paroli, and R. Pozzoli, in Non-Neutral Plasma Physics VIII, p. 273, AIP Conference Proceedings 1521, ed. by X. Sarasola, L. Schweikhard and T. S. Pedersen (AIP, New York, 2013).

[5] M. Maggiore, M. Cavenago, M. Comunian, F. Chirulotto, A. Galatà, M. De Lazzari, A. M. Porcellato, C. Roncolato, S. Stark, A. Caruso, A. Longhitano, F. Cavaliere, G. Maero, B. Paroli, R. Pozzoli, and M. Romé, Rev. Sci. Instrum. 85, 02 B909 (2014).

[6] C. F. Driscoll and K. S. Fine, Phys. Fluids B 2, 1359 (1990).

[7] M. Romé and F. Lepreti, Eur. Phys. J. Plus 126, 38 (2011).

[8] B. M. Jelenkovi, A. S. Newbury, J. J. Bollinger, T. B. Mitchell, and W. M. Itano, Nucl. Instrum. Meth.
B 192, 117 (2002).

[9] P. J. Morrison, in Mathematical Methods in Hydrodynamics and Integrability in Related Dynamical Systems, p. 13, AIP Conference Proceedings 88, ed. by M. Tabor and Y. Treve (AIP, New York, 1982).

[10] P. J. Morrison, Phys. Lett. 80A, 383 (1980).

[11] J. E. Marsden and A. Weinstein, Physica D 4, 394 (1982).

[12] P. J. Morrison, E. Tassi, and C. Tronci, in Nonlinear Physical Systems Spectral Analysis, Stability and Bifurcation, p. 311, ed. by O. N. Kirillov and D. E. Pelinovsky (Wiley and Sons, Hoboken, 2013).

[13] C. Tronci, J. Phys. A: Math. and Theor. 43, 375501 (2010).

[14] D. D Holm and C. Tronci, Comm. Math. Sci. 10, 191 (2012).

[15] M. D. Kruskal and C. Oberman, Phys. Fluids, 1, 275 (1958).

[16] V. I. Arnold, Doklady Mat. Nauk. 162, 773 (1965).

[17] P. J. Morrison, Rev. Mod. Phys. 70, 467 (1998).

[18] D. D. Holm, J. E. Marsden, T. Ratiu, and A. Weinstein, Phys. Rep. 123, 1 (1985).

[19] G. E. Morfill and A. V. Ivlev, Rev. Mod. Phys. 81, 1353 (2009).

[20] C. S. Gardner, Phys. Fluids 6, 839 (1963).

[21] R. C. Davidson and S. M. Lund, Phys. Plasmas 3, 2540 (1991).

[22] D. H. E. Dubin and T. M. O'Neil, Rev. Mod. Phys. 71, 87 (1999). 